\title{
La diversidad cognitiva como criterio de selección de equipos de aprendizaje cooperativo
}

\section{Cognitive diversity as criterion for the selection of cooperative learning teams}

\author{
Raúl Adolfo Pereira Nuñez ${ }^{(1)}$, Graciela Inés Núñez Benavidez ${ }^{(1)}$, \\ José Antonio Naranjo Rodríguez ${ }^{(2)}$
}

(1) Universidad Nacional de San Juan, Argentina

(2) Universidad de Granada

Resumen: Se exponen resultados de la indagación sobre la utilización de la diversidad cognitiva como criterio de selección para constituir equipos de trabajo, en el Modelo de Aprendizaje Cooperativo. La diversidad cognitiva se determinó con la interpretación que realizaron los estudiantes basándose en la Naturaleza Corpuscular de la Materia. Se presentan resultados obtenidos con equipos homogéneos y heterogéneos conformados con distinto criterio. La muestra estuvo constituida por alumnos universitarios de tercer año del Profesorado en Química. Los resultados alcanzados muestran que la heterogeneidad cognitiva puede ser utilizada como un criterio válido para la formación de equipos cooperativos.

Palabras clave: aprendizaje cooperativo, diversidad cognitiva, ciencias naturales.

Abstract: Results from the inquiry about the use of cognitive diversity as criterion for the selection to form work teams within the Model of Cooperative Learning are presented. Cognitive diversity was determined from the interpretation done by students about the Corpuscular Nature of Matter. Results obtained with homogeneous and heterogeneous teams, formed from different criteria are presented. The sample was formed by third year university students attending the career of University Teacher of Chemistry. The results show that cognitive heterogeneity can be used as a valid criterion for the formation of cooperative teams.

Keywords: cooperative learning, cognitive diversity, natural sciences

(Fecha de recepción: noviembre, 2016, y de aceptación: mayo, 2017)

DOI: 10.7203/DCES.32.9214 


\section{Introducción y objetivo}

Con este trabajo pretendemos revalorizar el aprendizaje cooperativo en el ámbito universitario y específicamente en la enseñanza de las Ciencias de la Naturaleza.

La enseñanza de las Ciencias de la Naturaleza se encuentra con diversos problemas originados por distintas causas, entre las que podemos mencionar la existencia de una marcada heterogeneidad cognitiva en los alumnos, referida, entre otros aspectos, a distintos niveles de conocimientos, información, desarrollo de capacidades y fundamentalmente en los modos de aprender de los estudiantes. En general, en la metodología tradicional empleada en la enseñanza de estas ciencias, la heterogeneidad cognitiva no es tenida en cuenta y por lo tanto no se obtienen los resultados esperados. Sin embargo, la diversidad en el aula es una característica que según diversos autores (Abad y Benito, 2006; CEJRO, 2016; Riera Romero, 2011; Soriano y Begoña Vigo, 2008) potencia el aprendizaje cuando se utiliza una metodología cooperativa.

La investigación educativa muestra un gran interés en encontrar una forma de enseñanza que responda al reconocimiento de la existencia de la diversidad en la forma en que los alumnos aprenden, a pesar de tener la misma edad, similar entorno social y educativo y muchos otros aspectos en común. En este sentido debemos reconocer las diversas actitudes de los estudiantes frente al objeto de conocimiento, entre las que se puede mencionar que algunos lo hacen solos y otros en compañía de pares; escuchando, practicando o reflexionando con mayor o menor velocidad. Atender a la diversidad del aula implica asumir la factibilidad de la conformación de clases donde coexistan las realidades curriculares junto a las del alumnado. Esta afirmación nos desafía y nos apremia a trabajar, reflexionar y cambiar continuamente para diagnosticar la diversidad y potenciarla en beneficio del aprendizaje. Al respecto, concordamos con Mateo Sánchez (2005), en que atender a la diversidad es algo más que un problema normativo de la ley educativa en vigencia, supone más bien una máxima de la pedagogía y del sentido común. La importancia de la diversidad cognitiva, en la conformación de equipos de aprendizaje cooperativo, constituye una realidad a tener en consideración. Nos preguntamos, entonces, ¿se puede adoptar como criterio de conformación de equipos de aprendizaje cooperativo la diversidad cognitiva? Este cuestionamiento trataremos de dilucidarlo en esta investigación.

El objetivo de este trabajo es determinar si la utilización de la diversidad cognitiva (determinada a partir de las interpretaciones que realizan los estudiantes sobre el fenómeno de disolución, utilizando la naturaleza corpuscular de la materia) constituye un criterio válido en la conformación de equipos de aprendizaje cooperativo.

\section{Marco teórico}


Aprendizaje cooperativo y el proceso de enseñanza

La cooperación consiste en trabajar juntos para alcanzar objetivos comunes. En una situación cooperativa, los individuos procuran obtener resultados que sean beneficiosos para ellos mismos y para los demás miembros del equipo. El aprendizaje cooperativo consiste en el empleo didáctico de equipos reducidos, utilizando una estructura de la actividad tal que asegure la participación igualitaria y una máxima potenciación de la interacción simultánea en los alumnos para maximizar su propio aprendizaje y el de los demás (Johnson, Johnson y Smith, 2014; Kagan, 2014). El estudio o aprendizaje cooperativo es una técnica instruccional que se caracteriza por el trabajo en grupos pequeños de los alumnos en el desarrollo de una tarea estructurada. (Madrid, Arellano, Jara, Merino y Balochi, 2013)

El aprendizaje cooperativo da la oportunidad de enseñar y aprender en cooperación, es decir, realza el aprendizaje que se da entre los estudiantes. La instrucción no sólo viene de parte del profesor, sino que recae en ellos como participantes activos en el proceso. La estrategia propone que el alumno, al ser parte de un equipo del cual depende su desempeño, asegurará que los otros integrantes del equipo también tengan un buen desempeño. Este tipo de aprendizaje recae en una enseñanza compartida, son los propios estudiantes los que jugarán roles como ayudantes o tutores. Este enfoque cambia la función del profesor instructor a un profesor asesor de equipos de trabajo cooperativo y él es responsable de crear las estructuras que llevarán al aprendizaje cooperativo.

Las técnicas de aprendizaje cooperativo constituyen una metodología innovadora que puede ayudar a resolver algunos problemas en el ámbito educativo, como el fracaso escolar, la falta de motivación, las relaciones profesores alumno, el maltrato entre iguales y, por supuesto, el tratamiento de la multiculturalidad en el aula. (León, Felipe, Iglesias y Latas, 2011).

El aprendizaje cooperativo es cada vez más utilizado en la enseñanza, dado que posibilita la utilización de problemáticas reales para ser resueltas en función de fundamentos teóricos y a la vez contribuye a la disminución de la ansiedad y la frustración que pueden producir los exámenes, pues la responsabilidad de los resultados es compartida por los miembros del equipo (Durán Aponte y Durán García, 2013). Entre las características sobresalientes del aprendizaje cooperativo, Jiménez García (2011) señala la existencia de:

- un objetivo común,

- una relación de igualdad entre todos los miembros,

- una relación de interdependencia positiva entre los miembros del equipo,

- una relación de cooperación, de ayuda y de exigencia mutua y

- la existencia de un vínculo afectivo. 
Mas allá de los resultados obtenidos en cuanto a rendimiento, el trabajo cooperativo proporciona una situación de aprendizaje más dinámica y atractiva, otorgando al alumno más responsabilidad y poder sobre su propio aprendizaje, aumentando su percepción de autonomía y competencia (León y otros, 2011). Además, García Almiñana y Amante García (2006) afirman que el aprendizaje cooperativo ofrece posibilidades de potenciación del nivel de conocimientos adquiridos y de mejora del resultado global del trabajo. Por otra parte, estos autores, destacan ventajas del mismo relacionadas con mejores actitudes de implicación y de iniciativa y con el incremento de la motivación del estu- diante por la tarea, de la comprensión de lo que se hace y del porqué se hace, del volumen de trabajo realizado, de la calidad del mismo, del grado de dominio de procedimientos y conceptos y de la relación social. Por lo que consideramos que estos beneficios justifican ampliamente su uso.

En el ámbito educativo se pueden contextualizar tres tipos de relaciones sociales, según el logro de objetivos y el tipo de recompensa que reciben los individuos por su trabajo: competición, individualización y cooperación (Johnson y Johnson, 1999). En el siguiente cuadro se muestran algunas diferencias entre ellas, referidas al aprendizaje:

\section{Cuadro I}

\section{Comparación entre aprendizaje cooperativo, competitivo e individualista}

Fuente: elaboración propia

\begin{tabular}{|c|c|c|c|}
\hline & $\begin{array}{l}\text { Aprendizaje } \\
\text { Cooperativo }\end{array}$ & $\begin{array}{l}\text { Aprendizaje } \\
\text { Competitivo }\end{array}$ & $\begin{array}{l}\text { Aprendizaje } \\
\text { Individualista }\end{array}$ \\
\hline Objetivo & $\begin{array}{l}\text { Los alumnos } \\
\text { trabajan juntos en } \\
\text { equipos reducidos } \\
\text { para maximizar } \\
\text { su aprendizaje y } \\
\text { el de los demás. }\end{array}$ & $\begin{array}{l}\text { Los alumnos } \\
\text { trabajan } \\
\text { comparándose con } \\
\text { los demás y con el } \\
\text { empeño de lograr la } \\
\text { máxima distinción. }\end{array}$ & $\begin{array}{l}\text { Los alumnos trabajan } \\
\text { con independencia, } \\
\text { a su propio ritmo } \\
\text { para lograr metas } \\
\text { de aprendizaje } \\
\text { personales, } \\
\text { desvinculadas de las } \\
\text { de los demás alumnos. }\end{array}$ \\
\hline $\begin{array}{l}\text { Esquema de } \\
\text { interacción }\end{array}$ & $\begin{array}{l}\text { Los estudiantes } \\
\text { estimulan el éxito } \\
\text { de los demás, se } \\
\text { escuchan y se } \\
\text { prestan ayuda. }\end{array}$ & $\begin{array}{l}\text { Los estudiantes } \\
\text { obstruyen el éxito } \\
\text { de los demás, } \\
\text { intentan disminuir } \\
\text { su rendimiento y se } \\
\text { niega a ayudarlos. }\end{array}$ & $\begin{array}{l}\text { Los estudiantes } \\
\text { trabajan } \\
\text { independientemente } \\
\text { y tienen libertad para } \\
\text { decidir si ayudan o no } \\
\text { a los otros. }\end{array}$ \\
\hline
\end{tabular}




\begin{tabular}{|l|l|l|l|}
\hline Aplicación & $\begin{array}{l}\text { Este tipo de } \\
\text { aprendizaje } \\
\text { es de amplia } \\
\text { aplicación. Se } \\
\text { puede promover } \\
\text { en cualquier } \\
\text { tarea, materia } \\
\text { o programa de } \\
\text { estudios. }\end{array}$ & $\begin{array}{l}\text { Este tipo de } \\
\text { aprendizaje presenta } \\
\text { limitaciones en } \\
\text { su aplicación con } \\
\text { relación a cuándo y } \\
\text { cómo emplearlo de } \\
\text { forma apropiada. }\end{array}$ & $\begin{array}{l}\text { Su aplicación } \\
\text { presenta igualmente } \\
\text { limitaciones. No todas } \\
\text { las tareas, materias } \\
\text { o cursos se prestan } \\
\text { para este tipo de } \\
\text { aprendizaje. }\end{array}$ \\
\hline Evaluación & $\begin{array}{l}\text { Se evalúa el } \\
\text { trabajo individual } \\
\text { del alumno y el } \\
\text { trabajo del equipo, } \\
\text { de acuerdo } \\
\text { con criterios } \\
\text { cognitivos y } \\
\text { actitudinales. }\end{array}$ & $\begin{array}{l}\text { Se evalúa el trabajo } \\
\text { de cada alumno } \\
\text { de acuerdo a una } \\
\text { norma basada } \\
\text { fundamentalmente } \\
\text { en el desempeño. }\end{array}$ & $\begin{array}{l}\text { Se evalúa con sus } \\
\text { propios criterios el } \\
\text { trabajo individual de } \\
\text { cada alumno. }\end{array}$ \\
\hline
\end{tabular}

En concordancia con Pere Pujolás Maset (2012), consideramos que una estructura individualista o competitiva de la actividad en el aula, no permite el desarrollo de algunas competencias básicas. Es la estructura cooperativa la que posibilita el enriquecimiento de competencias básicas como las comunicativas: expresar, argumentar e interpretar pensamientos, sentimientos $y$ hechos; escuchar ideas ajenas; aceptar y realizar críticas constructivas; colocarse en el lugar de otro de forma empática; respetar opiniones distintas a las propias con sensibilidad y espíritu crítico, y algunas competencias sociales como: practicar el diálogo y la negociación para resolver conflictos; trabajar en equipo aportando lo que uno sabe junto a lo que saben los demás para resolver juntos problemas comunes.

Además Pere Pujolás Maset (2012), reflexiona que trabajar en equipos cooperativos sobre contenidos curriculares de las distintas áreas, brinda a los alumnos la oportunidad y necesidad de practicar aquellas habilidades sociales propias del trabajo cooperativo. Por ejemplo se aprende a:

- exponer el propio punto de vista

- buscar argumentos y defender el punto vista

- aceptar el punto de vista de otro

- buscar distintas alternativas a la solución de un problema

- decidir la mejor alternativa entre todos 
- pensar sobre los propios actos, los del equipo y los de la clase

- contrastar los actos propios, del equipo y de la clase con normas consensuadas previamente

- identificar aciertos propios y ajenos

- identificar y reconocer errores

- hablarse claro y con respeto y

- regular el comportamiento en trabajo en equipo.

En CEJRO (2012) se afirma que dentro de las dinámicas cooperativas los alumnos ponen en práctica estrategias superiores a las utilizadas por los estudiantes en condiciones competitivas o individualistas. El proceso de discusión en los equipos cooperativos fomenta la utilización de estrategias cognitivas de aprendizaje de más calidad que en los casos de razonamiento individual que se generan en situaciones competitivas $\mathrm{o}$ individualistas.

De acuerdo con Fernández Batanero (2012), adquirir hábitos y destrezas para el aprendizaje autónomo y cooperativo y promoverlo entre los estudiantes constituye una competencia básica del profesor. Desde el punto de vista de la investigación, en el marco de la legislación educativa y de las competencias profesionales, se considera pertinente la preparación de los futuros docentes para la atención a la diversidad desde la colaboración y la reflexión compartida (Soriano y Vigo, 2008).

El aprendizaje cooperativo, a partir de las alternativas expuestas, es la metodología más efectiva, pero al mismo tiempo la menos utilizada en las aulas universitarias (León, Felipe, Iglesias y Latas, 2011). El aprendizaje individual en el aula sigue siendo el tipo de interacción más difundido a todos los niveles educativos y sobre todo en las aulas universitarias. Mediante este acercamiento clásico a la enseñanza el profesor se dirige por igual a todos los alumnos y resuelve individualmente las dudas o los problemas que van surgiendo en ellos, de manera que cada alumno se centre únicamente en sus tareas para lograr resultados positivos. La consecución de los objetivos individuales no influye por lo tanto en los resultados de los demás y la recompensa viene determinada por el trabajo personal sin prever ningún tipo de interacción y sin tener en cuenta el trabajo de los compañeros de clase (Lazzari, 2014).

La enseñanza universitaria está más bien dominada por una combinación de prácticas competitivas e individualistas del tipo dictado de clase y evaluación a partir de criterios de excelencia. La aplicación del aprendizaje cooperativo en el contexto universitario, puede dar cauce al propósito de incluir metodologías docentes activas e innovadoras. Así, con la finalidad de favorecer la calidad educativa, el reto del profesor universitario consiste en integrar procesos de investigación, innovación y evaluación de su práctica docente. En definitiva, el aprendizaje cooperativo se convierte en un indicador estimable y predictor de buenos resultados. 
El aprendizaje cooperativo y el colaborativo son enfoques que en su forma son similares, ambos trabajan con equipos de alumnos, no obstante, características tales como el objetivo que persiguen, las estructuras o el rol del profesor los diferencian. Panitz (2001), plantea que éstas se pueden sintetizar en que el aprendizaje cooperativo tiene como finalidad la construcción de nuevas ideas con la contribución de pares, esto favorece especialmente a los estudiantes que tienen mayores dificultades y enriquece a los aventajados. El aprendizaje colaborativo tiene como objetivo que cada estudiante haga su mejor aporte a un fin común, a partir del desarrollo de nuevas ideas, lo que no necesariamente involucra a aquellos estudiantes con dificultades de aprendizaje. Respecto de la función del profesor, en el aprendizaje cooperativo es el que propone el problema y determina el rol de cada alumno para su abordaje, es decir cada estudiante se responsabiliza de una parte de la solución de la tarea. En el aprendizaje colaborativo el profesor propone la actividad, se transforma en guía y son los propios estudiantes los que determinan sus roles.

De manera indistinta, en el aprendizaje cooperativo, suele utilizarse los términos grupo y equipo con un significado similar, sin embargo resulta conveniente establecer alguna diferenciación. En este sentido Gómez Mujica y Acosta Rodríguez (2003), plantean que se entiende por grupo a un conjunto de personas reunidas en torno a un núcleo o eje, que persiguen objetivos, intereses o acciones comunes, y necesitan unas de otras para alcanzar objetivos específicos. Así, podemos identificar grupos sociales primarios y secundarios. El primer grupo social al que pertenecemos desde el momento que nacemos, no es otro que la familia. Es donde forjamos nuestra personalidad, adquirimos las primeras habilidades y comenzamos el proceso de inserción social a través del núcleo familiar. La escuela también constituye un grupo social primario, en cuanto establecemos, en general y dependiendo de las circunstancias, lazos duraderos con nuestros compañeros o algunos de ellos. Al hablar de grupos distinguimos un conjunto de personas cohesionadas hacia un fin común, con organización y compromiso. Cuando ese grupo alcanza su meta más avanzada de nivel de sistematización, solidaridad, cooperación, motivación, organización y estabilidad se transforma en equipo. Todo equipo es un grupo, pero no todo grupo es un equipo, en el equipo se evidencia la solidaridad y la cooperación.

Según lo sugerido por Johnson, Johnson y Smith (2014), es el docente el que debe decidir con el tipo de equipo que trabajará. Estos equipos de aprendizaje deberán ser homogéneos o heterogéneos. Por lo general, son preferibles los equipos heterogéneos, compuestos por estudiantes con diferentes rendimientos y distintos intereses, aunque a veces es conveniente emplear equipos homogéneos, cuando los miembros tienen similar capacidad para enseñar determinadas prácticas sociales o 
alcanzar ciertos objetivos conceptuales. Trabajar con equipos heterogéneos permite que los alumnos tengan acceso a diversas perspectivas y métodos de resolución de problemas y produce mayor desequilibrio cognitivo, necesario para estimular el aprendizaje y el desarrollo intelectual de los alumnos. Los equipos heterogéneos tienden a promover un pensamiento más profundo, un mayor intercambio de explicaciones y una mayor tendencia a asumir puntos de vista durante los análisis del material, todo lo cual incrementa la comprensión, el razonamiento y la retención a largo plazo.

En la implementación del modelo de aprendizaje cooperativo, se han utilizado diversas formas de constitución de equipos. Johnson, Johnson y Holubec (1999) consideran las siguientes:

- Equipos seleccionados por los propios alumnos: los estudiantes se agrupan libremente.

- Equipos seleccionados por el docente: el profesor decide quién va a trabajar con quién.

- Equipos seleccionados al azar: distribución aleatoria. Este tipo de constitución de equipos presenta las siguientes variaciones:

El método matemático: se propone a los alumnos un problema matemático y se les pide que (a) resuelvan el problema, (b) encuentren compañeros con la misma respuesta y (c) formen un equipo.
Personajes históricos: se les entrega a los estudiantes una ficha con el nombre de algún personaje histórico y se les pide que se agrupen con los compañeros que tengan personajes del mismo período histórico.

Personajes literarios: se les entrega a cada alumno una ficha con el nombre de algún personaje de obras literarias leídas en clase y se les pide que se agrupen en función de los personajes que pertenecen a la misma obra.

- Equipos seleccionados de forma estratificada: es un procedimiento similar al anterior, con la salvedad de que el docente se asegura de que en cada equipo haya uno o dos alumnos con ciertas características específicas (como determinado nivel de lectura, estilo de aprendizaje, orientación a la tarea o interés personal).

Sobre la selección en forma estratificada Slavin (1999) plantea el sistema de distribución según rendimiento que consiste en:

1. Clasificar a los alumnos, en una lista según su desempeño previo.

2. Determinar la cantidad de equipos, dividiendo entre 4 el total de alumnos. Si la división no es exacta, deberá decidirse entre una combinación 4-3 ó 4-5.

3. Formar los equipos equilibrándolos de tal modo que cada uno esté 
integrado por alumnos con niveles alto, medio y bajo, de manera tal que el nivel de desempeño promedio de todos los equipos sea más o menos igual. Si los equipos no están distribuidos de forma pareja, se intenta lograr el equilibrio moviendo alumnos de niveles de desempeño parecidos pero de diferente equipo étnico, sexo o nivel de integración.

\section{Metodología}

La metodología de la investigación consistió en:

- Aplicación individual de un diagnóstico sobre la naturaleza corpuscular de la materia (NCM) (prestest).

- Trabajo cooperativo sobre vitamina C, con cuatro equipos: dos constituidos basados en la diversidad cognitiva (homogéneo y heterogéneo) y los otros dos (homogéneo y heterogéneo) en base a la distribución estratificada propuesta por Slavin (1999).

- Aplicación individual del diagnóstico sobre NCM (postest).

- Comparación de los resultados obtenidos por los estudiantes en pre y postest.

\section{Selección de la muestra de estudiantes}

La muestra se constituyó con 16 estudiantes universitarios, cuyas edades oscilan entre los 19 y 31 años, perte- necientes al tercer año del Profesorado en Química de una Universidad estatal.

\section{Diagnóstico de las interpretaciones individuales en el área de la NCM}

Para constituir los equipos en base a la diversidad cognitiva se aplicó un diagnóstico y se eligió trabajar en el área de la naturaleza corpuscular de la materia (NCM). Esta elección se fundamenta en que es un contenido muy importante para la ciencia actual y es uno de los principales objetivos educativos de la mayor parte de los currículos de ciencias de los cursos superiores de la enseñanza básica. Además, se destaca su poder explicativo, su potencialidad para favorecer el trabajo con modelos físicos y su uso como herramienta válida para clarificar confusiones de conceptos macroscópicos. Karacop y Doymus (2013), señalan que a través de las técnicas de animación y rompecabezas del aprendizaje cooperativo, los estudiantes tuvieron mayor éxito en la comprensión de la NCM.

Se realizó una búsqueda de instrumentos validados que permitieran delimitar las interpretaciones individuales. Se eligió del diagnóstico aplicado por Benarroch, Benarroch (2000), las tareas relacionadas con la disolución. Las mismas consisten en actividades con respuestas abiertas y representaciones gráficas elaboradas por los estudiantes. Así se trabajó con: 
- Proceso de disolución de sólido granular.

- Dilución de solución de sólido granular.

- Efecto de la temperatura en la disolución de sólido granular.

Los resultados se analizaron de la siguiente forma:

a. Transcripción literal de las respuestas de los estudiantes para cada actividad.

b. Análisis de las explicaciones e ilustraciones realizadas por los estudiantes, ante las distintas situaciones planteadas.

c. Valoración de las interpretaciones individuales.

d. Distribución de los estudiantes.

e. Formación de equipos.

Este diagnóstico se aplicó nuevamente a los equipos, luego de realizadas las actividades de aprendizaje, en instancia de postest.

\section{Formación de equipos}

Equipos $\mathrm{A}(\mathrm{He})$ y $\mathrm{B}(\mathrm{Ho})$ constituidos basándose en la diversidad cognitiva: Los estudiantes respondieron el diagnóstico para determinar las interpretaciones individuales en el área de la NCM. En el Cuadro II.(Puntajes adjudicados a las interpretaciones), se detallan los criterios seguidos para la asignación de puntajes. Teniendo en cuenta los resultados (diversidad cognitiva) se conformaron dos equipos, uno heterogéneo, $\mathrm{A}(\mathrm{He})$, (integrado por dos estudiantes que realizaron las interpretaciones más cercanas a las científicas y dos alumnos que manifestaron las más alejadas) y otro homogéneo, $\mathrm{B}(\mathrm{Ho})$, con los cuatro restantes, cuyas interpretaciones fueron intermedias. Es de destacar que la agrupación de los estudiantes por su nivel cognitivo puede variar sustancialmente según la prueba realizada.

Con el objeto de comparar los resultados de los equipos se realizó una modificación en la distribución estratificada propuesta por Slavin (1999) que consistió en integrar los equipos como se detalla a continuación:

Equipos $\mathrm{C}(\mathrm{He})$ y $\mathrm{D}(\mathrm{Ho})$ constituidos utilizando la distribución estratificada propuesta por Slavin (1999), modificada teniendo en consideración los siguientes criterios:

a. Entrevista con docentes de las cátedras de asignaturas cursadas con anterioridad (Química General, Química Orgánica Alifática, Química Inorgánica, Cinética y Termodinámica Química). Los datos de las entrevistas se registraron en una lista de cotejo que incluía características personales de los estudiantes y su participación en clases teóricas, prácticas y de laboratorio. Estas variables fueron ponderadas.

b. Promedio de las calificaciones obtenidas en las evaluaciones de cada una de las asignaturas 
y posteriormente el promedio general incluyendo todas.

Con las ponderaciones obtenidas a partir de la aplicación de estos criterios, se procedió de la siguiente manera:

- Se estableció un orden creciente de calificaciones de los estudiantes, teniendo en cuenta los criterios a) y b).

- Se formó el equipo heterogéneo eligiendo dos alumnos del orden superior y dos del inferior.

- Se formó el equipo homogéneo con los cuatro estudiantes de orden medio.

Metodología de aplicación del Modelo de Aprendizaje Cooperativo

La metodología seguida consistió en la adaptación de las etapas previstas en el Modelo de Aprendizaje Cooperativo, División de la Clase en Equipos de Aprendizaje (DCGA) ${ }^{1}$, mencionado en Eggen y Kauchak (2012). A continuación se detallan las etapas realizadas:

1. Enseñanza: El docente explicó las actividades a realizar y distribuye las guías de actividades.

2. Transición al trabajo en equipos: Conformados los equipos, el docente determinó el rol de cada alumno en la aplicación del Modelo Cooperativo.
3. Estudio en equipo y monitoreo: El docente controló el cumplimiento de la participación y de los roles asignados a los alumnos.

4. Pruebas: Aplicación de postest.

5. Reconocimiento de logros: Asignación de puntajes individual y grupal.

Actividades de aprendizaje realizadas por los equipos

Como material didáctico para la aplicación del Modelo de Aprendizaje Cooperativo, se utilizó una guía con actividades, bibliografía e información que facilitara la recogida de datos de los estudiantes. El contenido elegido fue la disolución de la vitamina C. El trabajo de los alumnos se centró en la interpretación, de acuerdo a la NCM, de la disolución de una pastilla de vitamina $\mathrm{C}$.

Descripción de la evaluación de los resultados

Ésta se basó en la evaluación que propone Slavin (1999). Se realizó el análisis del desempeño individual y grupal de los estudiantes, en situación de pretest y postest. Los resultados obtenidos en situación de pretest en las actividades sobre NCM se utilizaron para la conformación de dos equipos y además consti-

\footnotetext{
${ }^{1}$ En inglés STAD (Students Teams Achievement Division)
} 
tuyeron los puntajes iniciales de todos los equipos. Posteriormente se realizó la intervención didáctica, mediante el trabajo cooperativo, para luego evaluar la evolución individual en la interpretación de la NCM, en situación de postest.
En esta investigación, cuando se evaluaron las interpretaciones de los estudiantes sobre los fenómenos que se les presentaron, se adjudicaron los puntajes que surgen de la siguiente escala de valoración:

\section{Cuadro II. Puntajes adjudicados a las interpretaciones}

Fuente: elaboración propia

\begin{tabular}{|l|c|}
\hline \multicolumn{1}{|c|}{ Interpretación científica de la NCM } & Puntaje \\
\hline Plena (interacciones, vacío, distancias intermoleculares, movimiento) & 90 \\
\hline Semi plena (vacío, distancias intermoleculares, movimiento) & 80 \\
\hline Cercana (movimiento) & 70 \\
\hline Intermedia (solo pequeñas esferas) & 60 \\
\hline Confusa (mezcla de modelo continuo y corpuscular) & 50 \\
\hline Leve (representaciones con puntos) & 40 \\
\hline Sin interpretación (modelo continuo) & 30 \\
\hline
\end{tabular}

Puntos de superación: Los puntos de superación son recompensas sobre la base del desempeño de cada estudiante en postest, comparados con los puntos del pretest. En este trabajo se tuvo en cuenta tal recompensa en las calificaciones de los estudiantes, en la tarea desarrollada.

En general, los métodos de aprendizaje cooperativo tienen una característica común y es el concepto de igualdad de oportunidades para el logro del éxito, lo que significa que todos los estudiantes más allá de su desempeño individual anterior, pueden esperar logros de acuerdo con sus esfuerzos. Esto se traduce, en la valoración, recompensando, además, a los estudiantes con cinco puntos de bonificación, si el puntaje del equipo en postest es diez puntos mayor que en pretest. (Eggen y Kauchack, 2012).

A continuación se muestra la forma de recompensar los puntos de superación: 


\section{Cuadro III. Puntos de superación}

Fuente: Eggen y Kauchack, 2012

\begin{tabular}{|l|c|}
\hline \multicolumn{1}{|c|}{ Puntaje obtenido en postest } & Ptos de superación \\
\hline Debajo del puntaje del pretest & 0 \\
\hline De uno a diez sobre el puntaje del pretest & 10 \\
\hline De diez a veinte sobre el puntaje de pretest & 20 \\
\hline $\begin{array}{l}\text { Más de veinte puntos sobre el puntaje de pretest o prueba } \\
\text { perfecta sin considerar el puntaje de pretest }\end{array}$ & 30 \\
\hline
\end{tabular}

\section{Resultados}

Integración de los equipos de trabajo

A continuación se describe cómo se determinaron los cuatro grupos de estudiantes que integraron la muestra.

Constitución de equipos en base al análisis de la diversidad cognitiva

$\mathrm{El}$ análisis de las interpretaciones de los estudiantes universitarios, ha puesto de manifiesto un espectro de resultados muy variado. De acuerdo con el desempeño para interpretar los fenómenos aplicando la NCM, se constituyeron los siguientes equipos con sus respectivos puntajes:

Equipo Heterogéneo A(He): $\mathrm{MaG}$ (40), EuG (50), FlS (80), GaV (80).

Equipo Homogéneo B(Ho): CiV (60), $\mathrm{MaH}$ (60), ViH (70), VaO (70).

Constitución de equipos en base a la distribución estratificada
A continuación se muestra los integrantes de los equipos $\mathbf{C}(\mathbf{H e})$ y $\mathbf{D}(\mathbf{H o})$, constituidos de la forma descripta en el punto 3.2:

Equipo Heterogéneo C(He): VaM (9), $\mathrm{SiR}(8), \mathrm{EmP}(5)$ y CeV (4).

Equipo Homogéneo D(Ho): $\mathrm{SiB}(7,5)$, PaM (7), RoE (6,5), $\operatorname{ErV~(6).~}$

\section{Trabajo Cooperativo}

A continuación se exponen los resultados alcanzados por los estudiantes en su trabajo individual y en su desempeño en el trabajo cooperativo, para los cuatro equipos.

\section{Desempeño individual y en equipo}

En las siguientes tablas se muestran los puntajes de las interpretaciones individuales sobre la NCM, alcanzados en pre y postest, la diferencia entre ambos y los puntos de superación correspondientes y la puntuación final (incluyendo los puntos de superación correspondientes). 
Resultados alcanzados por los equipos conformados a partir de la diversidad cognitiva

Equipo A(He):

Cuadro IV

Puntajes de las interpretaciones individuales y promedios de $\mathbf{A}(\mathrm{He})$

\begin{tabular}{|c|c|c|c|c|}
\hline NOMBRE & PRETEST & POSTEST & DIFERENCIA & PTOS SUP. \\
\hline MAG & 40 & 80 & 40 & 30 \\
\hline EuG & 50 & 80 & 30 & 20 \\
\hline GaV & 80 & 90 & 10 & 10 \\
\hline FlS & 80 & 90 & 10 & 10 \\
\hline Promedio & $\mathbf{6 2 , 5 0}$ & $\mathbf{8 5}$ & $\mathbf{2 2 , 5 0}$ & $\mathbf{1 7 , 5 0}$ \\
\hline
\end{tabular}

En la descripción de la evaluación de las pruebas, se especificó la posibilidad de adjudicar 5 puntos de bonificación, a cada integrante si el puntaje total del equipo iguala o supera en 10 la diferencia entre pre y postest. Basados en esta consideración, a cada integrante de este equipo le corresponden los 5 puntos de bonificación, obteniéndose los puntajes de la tabla V.
Cuadro V. Puntaje individual final considerando los puntos de bonificación del equipo $\mathrm{A}(\mathrm{He})$

\begin{tabular}{|c|c|}
\hline NOMBRE & PJE FINAL \\
\hline MaG & 85 \\
\hline EuG & 75 \\
\hline GaV & 95 \\
\hline FIS & 95 \\
\hline Promedio & $\mathbf{8 7 , 5 0}$ \\
\hline
\end{tabular}

Equipo B (Ho):

Cuadro VI. Puntajes de las interpretaciones individuales y promedio de equipo $B(\mathrm{Ho})$

\begin{tabular}{|c|c|c|c|c|}
\hline NOMBRE & PRETEST & POSTEST & DIFERENCIA & PTOS SUP. \\
\hline CiV & 60 & 70 & 10 & 10 \\
\hline MaH & 60 & 70 & 10 & 10 \\
\hline ViH & 70 & 80 & 10 & 10 \\
\hline VaO & 70 & 80 & 10 & 10 \\
\hline Promedio & $\mathbf{6 5}$ & $\mathbf{7 5}$ & $\mathbf{1 0}$ & $\mathbf{1 0}$ \\
\hline
\end{tabular}


Resultados alcanzados por los equipos conformados utilizando la distribución estratificada

Equipo C (He):

\section{Cuadro VII}

Puntajes de las interpretaciones individuales y promedio de equipo $\mathrm{C}(\mathrm{He})$

\begin{tabular}{|c|c|c|c|c|}
\hline NOMBRE & PRETEST & POSTEST & DIFERENCIA & P PTOS SUP. \\
\hline VaM & 50 & 60 & 10 & 10 \\
\hline SiR & 60 & 80 & 20 & 30 \\
\hline EmP & 70 & 90 & 20 & 30 \\
\hline CeV & 60 & 90 & 30 & 20 \\
\hline Promedio & $\mathbf{6 0}$ & $\mathbf{8 0}$ & $\mathbf{2 0}$ & $\mathbf{2 0}$ \\
\hline
\end{tabular}

A este equipo también corresponde adjudicarle 5 puntos de bonificación a cada estudiante dado que la diferencia entre pre y postest del equipo supera los 10 puntos.
Cuadro VIII. Puntaje individual final considerando los puntos de bonificación del equipo $\mathrm{C}(\mathrm{He})$

\begin{tabular}{|c|c|}
\hline NOMBRE & PJE FINAL \\
\hline VaM & 65 \\
\hline SiR & 85 \\
\hline EmP & 95 \\
\hline CeV & 95 \\
\hline Promedio & $\mathbf{8 5}$ \\
\hline
\end{tabular}

Equipo D(Ho):

Cuadro IX. Puntajes de las interpretaciones individuales y promedio de equipo $\mathrm{D}(\mathrm{Ho})$

\begin{tabular}{|c|c|c|c|c|}
\hline NOMBRE & PRETEST & POSTEST & DIFERENCIA & PTOS SUP. \\
\hline SiB & 60 & 70 & 10 & 10 \\
\hline PaM & 60 & 70 & 10 & 10 \\
\hline ErV & 60 & 70 & 10 & 10 \\
\hline RoE & 70 & 80 & 10 & 10 \\
\hline Promedio & $\mathbf{6 2 , 5 0}$ & $\mathbf{7 2 , 5 0}$ & $\mathbf{1 0}$ & $\mathbf{1 0}$ \\
\hline
\end{tabular}


En los equipos Homogéneos no se adjudican puntos de bonificación, dado que la diferencia entre pre y postest en el equipo, no supera el valor de 10 .

A partir del análisis de los resultados obtenidos se observa que:

- La totalidad de los estudiantes, independientemente del grupo al que pertenece, mejoró significativamente su desempeño académico.

- Los estudiantes con bajos puntajes en pretest, correspondientes a grupos heterogéneos, MaG (cuadro VI), CeV (cuadro IX) aumentaron significativamente sus ren- dimientos. Obteniendo la mayor diferencia en puntaje entre pos y pretest, en los dos casos.

\section{Comparación de los resultados} alcanzados por los equipos

En el siguiente cuadro se comparan los avances alcanzados por los cuatro equipos de estudiantes. Los mismos pueden visualizarse mediante la diferencia entre pre y postest, por los puntos de superación de cada equipo y por el cálculo de la ganancia porcentual. La ganancia porcentual se ha calculado mediante la razón entre la diferencia y el puntaje obtenido en pretest.

\section{Cuadro VIII}

\section{Puntajes de los equipos y ganancia porcentual}

\begin{tabular}{|c|c|c|c|c|c|}
\hline EQUIPO & PRETEST & POSTEST & DIFERENCIA & PTOS SUP. & GANANCIA \% \\
\hline $\mathbf{A}(\mathbf{H e})$ & 62,50 & 85 & 22,50 & 20 & 36,00 \\
\hline $\mathbf{C}(\mathrm{He})$ & 60 & 80 & 20 & 20 & 33,33 \\
\hline$\underline{\mathbf{B}(\mathrm{Ho})}$ & 65 & 75 & 10 & 10 & 15,38 \\
\hline$\underline{\mathbf{D}(\mathbf{H o})}$ & 62,50 & 72,50 & 10 & 10 & 16,00 \\
\hline
\end{tabular}

Del análisis de las comparaciones, entre los equipos heterogéneos y homogéneos, se infiere que:

- Aumentan los puntajes en todos los equipos.

- El mayor aumento de puntaje es en los equipos heterogéneos.

- La mayor diferencia entre post y pretest ocurre en los equipos heterogéneos, aunque los equipos homogéneos también obtienen diferencia positiva entre pre y postest. No obstante es menor que en los equipos heterogéneos.

- Los equipos heterogéneos obtienen mayores puntos de superación que los equipos homogéneos.

- Los resultados obtenidos en los equipos heterogéneos selecciona- 
dos por diferentes metodologías, son aproximados.

- Los resultados obtenidos en los equipos homogéneos seleccionados por diferentes metodologías, son aproximados.

- La ganancia porcentual es mayor en los equipos heterogéneos.

\section{Conclusiones}

A partir del análisis de los resultados obtenidos por los estudiantes que constituyen los distintos equipos, se infiere:

1. La utilización de las interpretaciones de los alumnos en el área de la NCM, constituye una herramienta válida para conocer la diversidad cognitiva. Por lo tanto puede aplicarse como una metodología de selección de equipos heterogéneos de aprendizaje cooperativo. Además, al brindar la posibilidad de constatar la diversidad de interpretaciones de los alumnos permite adoptar una metodología de enseñanza atendiendo a las necesidades cognitivas individuales, mediante la adecuación de las intervenciones didácticas.

2. Comparando las modalidades de conformación de equipos utilizadas se comprueba que aquella que utiliza la diversidad cognitiva permite la conformación de equipos cooperativos con una alta potencialidad educativa, dado que considera las capacidades cognitivas de los alumnos.

3. Referido al trabajo cooperativo, se puede advertir la conveniencia de trabajar con equipos conformados a partir de la diversidad cognitiva, de esta manera los alumnos:

a. se exponen a ideas variadas, múltiples perspectivas y diferentes formas de enfrentamiento de situaciones problemáticas,

b. se involucran en un pensamiento más elaborado, dan y reciben más explicaciones y adoptan con más frecuencia puntos de vista personales para discutir el material. Todo esto aumenta la profundidad de la comprensión, la calidad del razonamiento y la precisión de retención a largo plazo.

4. Se convalida que el trabajo cooperativo constituye un ámbito idóneo para atender a la diversidad cognitiva, siendo los docentes quienes han de asumir las diferencias cognitivas como algo característico de su quehacer cotidiano.

Como conclusión final se puede afirmar que la diversidad cognitiva, determinada a partir de las interpretaciones de los estudiantes sobre fenómenos físicos, aplicando el modelo de NCM, en una metodología cooperativa, aporta mejoras en el aprendizaje del alumnado que se implica en él. 
La lógica de la heterogeneidad está en la base de un proceso de intervención educativa abierta a la diversidad, que responde a las demandas de todos los alumnos, porque reconoce y acepta las diferencias individuales. Considerar al alumno como partícipe genuino, gestor comprometido por su propio desarrollo y el del grupo, y al docente como mediador, facilitador y animador de los aprendizajes, demanda el conocimiento experto y la puesta en práctica de teorías y metodologías apropiadas. El docente debe ofrecer un ambiente de aprendizaje en el que aprendan alumnos con estilos diferentes y en el que la metodología se adapte al contexto del aula.

\section{Referencias bibliográficas}

ABAD, M.;BENITO, Ma L., Coord.(2006). Como enseñar junt@s a alumnosdiferentes: Aprendizaje cooperativo. Experiencias de atención a la diversidad para una escuela inclusiva. Zaragoza: Gobierno de Aragón.

BENARROCH BENARROCH,A. (2000). El desarrollo cognoscitivo de los estudiantes en el área de la naturaleza corpuscular de la materia. Enseñan$z a$ de las Ciencias, ${ }^{\circ}$ 18(2), 235-246.

CEJRO (2012). COOPERATI VA DE ENSEÑANZA JOSÉ RAMÓN OTERO. Propuesta para la implantación de una estructura cooperativa en el aula. Laboratorio de Innovación Educativa. 2-85.

CEJRO (2016). COOPERATI VA DE ENSEÑANZA JOSÉ
RAMÓN OTERO. Por qué hacemos Aprendizaje Cooperativo. Laboratorio de Innovación Educativa. 1-15.

DURÁN APONTE, E. y DURÁN GARCÍA, M. (2013). Aprendizaje cooperativo en la Enseñanza de Termodinámica: Estilos de Aprendizaje y Atribuciones Causales. Journal of Lear-ning Styles, vol.6, n¹1, 1-22.

EGGEN, P. y KAUCHAK, D. (2012). Estrategias Docentes. Enseñanza de contenidos curriculares y desarrollo de habilidades de pensamiento. México: Fondo de Cultura Económica.

FERNÁNDEZ BATANERO, J.M. (2012). Capacidades y competencias docentes para la inclusión en la educación superior. Revista de la Educación Superior, vol. 41, n 162, 9-24. Disponible en: http://www.redalyc.org/articulo.oa?id=60425033001 $[23$ de junio de 2016]

GARCÍA ALMIÑANA, D. y AMANTE GARCÍA, B. (2006). Comunicación de Congreso. Algunas experiencias de aplicación del aprendizaje cooperativo y del aprendizaje basado en proyectos. I Jornadas de Innovación Educativa. España, Escuela Politécnica Superior de Zamora.

GÓMEZ MUJICA, A. y ACOSTA RODRÍGUEZ, H. (2003). Acerca del trabajo en grupos o equipos. ACIMED, vol.11, $\mathrm{n}^{\circ} 6$. Disponible en http://scielo.sld.cu/scielo.phpscriptsci_arttextpidS102494352003000600011lngesnrmiso. [30 de junio de 2016] 
JIMÉNEZ GARCÍA, M. (2011). Aprendizaje Cooperativo II. Educainnova Magazine.

http://www.educainova.com/opencms/ export/sites/default/educainnova/ revista/REVISTA ENERO 2011. [21 de junio de 2016]

JOHNSON, D. y JOHNSON, R. (1999). Aprender Juntos y Solos. Buenos Aires: Aique.

JOHNSON, D.; JOHNSON, R. y HOLUBEC, E. (1999). El aprendizaje Cooperativo en el aula. Buenos Aires: Paidós.

JOHNSON, D.; JOHNSON, R. y SMITH, K. (2014). Cooperative learning: Improving university instruction by basing practice on validated theory. Journal on Excellence in College Teaching, vol. 25, n³, 85-118.

KAGAN, S. (2014). Kagan structures, processing, and excellence in college teaching. Journal on Excellence in College Teaching, vol. 25, $\mathrm{n}^{\circ} 3,119$ 138.

KARACOP, A. y DOYMUS, K. (2013), Effects of jigsaw cooperative learning and animation techniques on students' understanding of chemical bonding and their conceptions of the particulate nature of matter. Journal of Science Education \& Technology, n ${ }^{\circ} 22,186-203$. DOI: $10.1007 / \mathrm{s} 10956-$ 012-9385-9.

LAZZARI, M. (2014). Combinación de Aprendizaje Cooperativo e Individual en una Asignatura de Química de Materiales. Revista Formación
Universitaria, vol. 7, $\mathrm{n}^{\circ} 4,39-46$.

DOI:10.4067/S0718-50062014000400005.

LEÓN, B.; FELIPE, E.; IGLESIAS, D.

y LATAS, C. (2011). El aprendizaje cooperativo en. la formación inicial del profesorado de Educación Secundaria. Revista de Educación, $\mathrm{n}^{\circ} 354$, 715-729.

MADRID J.C., ARELLANO M., JARA R., MERINO C. y BALOCHI E. (2013). El aprendizaje cooperativo en la comprensión del contenido "disoluciones". Un estudio piloto. Educación química, $\mathrm{n}^{\circ} 24$ (núm. extraord. 2), 471-479.

DOI:10.1016/0187-893x(13)72515-1.

MATEO SÁNCHEZ, J. (2005). La atención a la diversidad en ciencias a través de materiales curriculares adaptados. Revista Eureka sobre Enseñanza y Divulgación de las Ciencias, vol. $2, \mathrm{n}^{\circ} 3,416-429$.

PANITZ, T. (2001). Collaborative versus Cooperative Learning: Comparing the Two Concepts which will Helps us Understand the Underling nature of Interactive learning. Cooperative Learning and College Teaching, vol 8, $\mathrm{n}^{\circ} 2$. Disponible en http://home.capecod.net/ tpanitz/tedsarticles/coopdefinition.htm. [15 de junio de 2016].

PERE PUJOLÁS MASET, P. (2012). Aulas inclusivas y aprendizaje cooperativo. Education Siglo XXI, vol. 30, $\mathrm{n}^{\mathrm{o}} 1,89-112$.

RIERA ROMERO, G. (2011). El aprendizaje cooperativo como metodología clave para dar respuesta a la diversi- 
dad del alumnado desde un enfoque inclusivo. Revista Latinoamericana de Educación Inclusiva, vol. 5, $\mathrm{n}^{\circ} 2$, 133-149.

SLAVIN, R. (1999). Aprendizaje Cooperativo: Teoría, Investigación y Práctica. Buenos Aires: Aique.

SORIANO, J. y BEGOÑA VIGO, B. (2008). La formación de maestros para la atención a la diversidad: evaluación de una experiencia de reflexión conjunta entre futuros maestros de distintas especialidades", en II Jornadas de Innovación Docente, Tecnologías de la Información y de la Comunicación e Investigación Educativa. Zaragoza: Universidad de Zaragoza. 